Check for updates

Cite this: Nanoscale Adv., 2019, 1, 3883

Received 2nd July 2019

Accepted 30th August 2019

DOI: $10.1039 / c 9 n a 00419 j$

rsc.li/nanoscale-advances

\section{Solvent influence on the surface morphology of P3HT thin films revealed by photoemission electron microscopy $\uparrow$}

\author{
Falk Niefind, Shubhangi Karande, Frank Frost, Bernd Abel (D) * and Axel Kahnt (D) *
}

\begin{abstract}
Only rigorous understanding of the relationship between the nanoscale morphology of organic thin films and the performance of the devices built from them will ultimately lead to design rules that can guide a structured development on the field of organic electronics. Despite great effort, unraveling the nanoscale structure of the films is still a challenge in itself. Here we demonstrate that photoemission electron microscopy can provide valuable insights into the chain orientation, domains size and grain boundary characteristics of P3HT films spun cast from different solvents at room as well as at elevated temperatures.
\end{abstract}

Minimizing the carbon footprint of mankind has emerged as a major challenge for the 21 st century. ${ }^{1}$ Successfully tackling climate change demands, among other things, decarbonisation of the energy sector. ${ }^{2-4}$ Here, organic semiconducting polymers can play an integral part as components of solar cells due to their mechanical flexibility and cheap production. ${ }^{5,6}$

Numerous techniques such as scanning near-field optical microscopy (SNOM), transmission electron microscopy (TEM), atomic force microscopy (AFM), near edge X-ray absorption fine structure (NEXAFS) spectroscopy, scanning transmission X-ray microscopy (STXM) and polarized X-ray scattering (PoXS) have been applied to unveil the morphology of these polymers. ${ }^{7-10}$ However, due to their semi-crystalline nature, it remains a great challenge to determine their structure. Consequently, this hinders the overall goal to correlate the morphology with the performance of the devices fabricated from these polymers. ${ }^{8}$

Recently photoemission electron microscopy (PEEM) has emerged as a powerful tool to investigate the P3HT thin film morphology. It has been demonstrated that by utilizing a polarized laser source in combination with a PEEM the

Leibniz Institute of Surface Engineering (IOM), Permoserstr. 15, 04318 Leipzig, Germany.E-mail: bernd.abel@iom-leipzig.de; axel.kahnt@iom-leipzig.de

$\dagger$ Electronic supplementary information (ESI) available: Experimental procedure, additional orientation maps, OPL plots, film thickness. See DOI: 10.1039/c9na00419j polymer chain orientation and the local degree of order can be revealed in a single, straightforward measurement. ${ }^{11}$

Furthermore we stress the fact that the non-destructive, tabletop PEEM measurements presented here offer an pathway to quickly screen different samples and to compare their respective performance, as well as the morphology to results gathered from other techniques. Owing to the nondestructive nature it is even possible to convert the samples investigated with PEEM into devices to obtain a direct correlation between the results obtained by PEEM and the device performance.

The morphology of organic films does strongly depend on the chosen solvent prior to spin coating. ${ }^{12-17}$ Therefore, being able to quickly image the nanoscale structure with PEEM and relate it to the respective solvent is a valuable enhancement in the field of organic electronics.

This is the first report investigating the effects different solvents have on the P3HT morphology with PEEM. We chose chlorobenzene (CB), 1,2-dichlorobenzene (DCB), 1,2,4-trichlorobenzene (TCB), thiophene (TP) and chloroform (CF) as solvents. They have previously been discussed as suitable solvents for P3HT in literature, see Table 1 for a selection of their properties.

Several groups have previously studied the effects of different solvents on the P3HT morphology or the respective device performance:

Table 1 Solubilities of P3HT in and boiling points of the respective solvents ${ }^{16}$

\begin{tabular}{lcc}
\hline Solvent & P3HT solubility $/ \mathrm{mg} \mathrm{mL}^{-1}$ & Boiling point $/{ }^{\circ} \mathrm{C}$ \\
\hline Trichlorobenzene & 2.7 & 214 \\
Chloroform & 14.1 & 60 \\
Dichlorobenzene & 14.7 & 180 \\
Chlorobenzene & 15.9 & 132 \\
Thiophene & 37.6 & 84
\end{tabular}


Bao et al. reported that the film quality and the field effect mobility in P3HT depend on the used solvent. ${ }^{12}$ They speculate that the solubility and the boiling point influence the film morphology. Especially the boiling point seems to be an apparent candidate, as solvents with a high boiling point evaporate more slowly. This increased evaporation period then grants the polymer chains more time to arrange in a crystalline structure.

Chang et al. picked up this line of argumentation: ${ }^{13}$ their best performing transistor was fabricated from a P3HT/TCB solution. The good solubility, but more importantly, the high boiling point of $214{ }^{\circ} \mathrm{C}$ of TCB is thought to lead to enhanced crystallization and thus to better performing devices.

By employing GIXD Yang et al. also reported that films prepared from solution with higher boiling point solvents usually exhibit a higher crystallinity. ${ }^{\mathbf{1 4}}$ However, they point out that the film prepared from the solution with the highest boiling point, shows the worst performance. This may indicate that other parameters have an equally high impact on the film quality and device performance. One parameter of particular interest is the polymer chain orientation, a quantity that we are able to measure with PEEM. ${ }^{11}$

In 2011 Dang et al. conclude that so far no clear correlation between the organic field-effect transistor OFET hole mobility in P3HT films and the solvent properties has been found in the literature. ${ }^{15}$ Consequently, additional research on this matter is advisable.

Machui et al. investigated the dependence of the $\mathrm{P} 3 \mathrm{HT}$ solubility in various solvents at different temperatures. ${ }^{\mathbf{1 6}}$ Their findings show that the solubility of P3HT does strongly depended on the respective temperature - the higher the temperature, the higher the solubility. Furthermore, they point out that the aggregates of the semi-crystalline P3HT are harder to dissolve than the amorphous parts. In case these aggregates are not fully dissolved by the solvent they could act as crystallization seeds during the spin coating process (vide infra).

In the following, we will show the effect that different solvents have on the P3HT thin film surface morphology.

We employ the aforementioned solvents to prepare two sets of samples: one spun cast from the solvent at room temperature (RT), the other set spun cast at elevated temperatures, about five centigrades below the boiling points. The results indicate that for the solvents investigated here the surface morphology, especially the domain size, does primarily depend on the solubility of P3HT in the particular solvent. The boiling point plays only a secondary role.

The left part of Fig. 1 displays the spatial distributions of the combination of the polymer-chain orientation and the degree of order in P3HT films spun cast from different solvents. A higher transparency in the images indicates a lower degree of order. The right side of Fig. 1 displays the images generated from films that were spun cast from a heated solution (for details see experimental section).

To compare the results from the different samples we employ the orientation persistence length (OPL). The OPL has been introduced before by Neff $e t$ al. and the reader is referred to the publication and the ESI $\uparrow$ for detailed insights. ${ }^{11}$ In short,

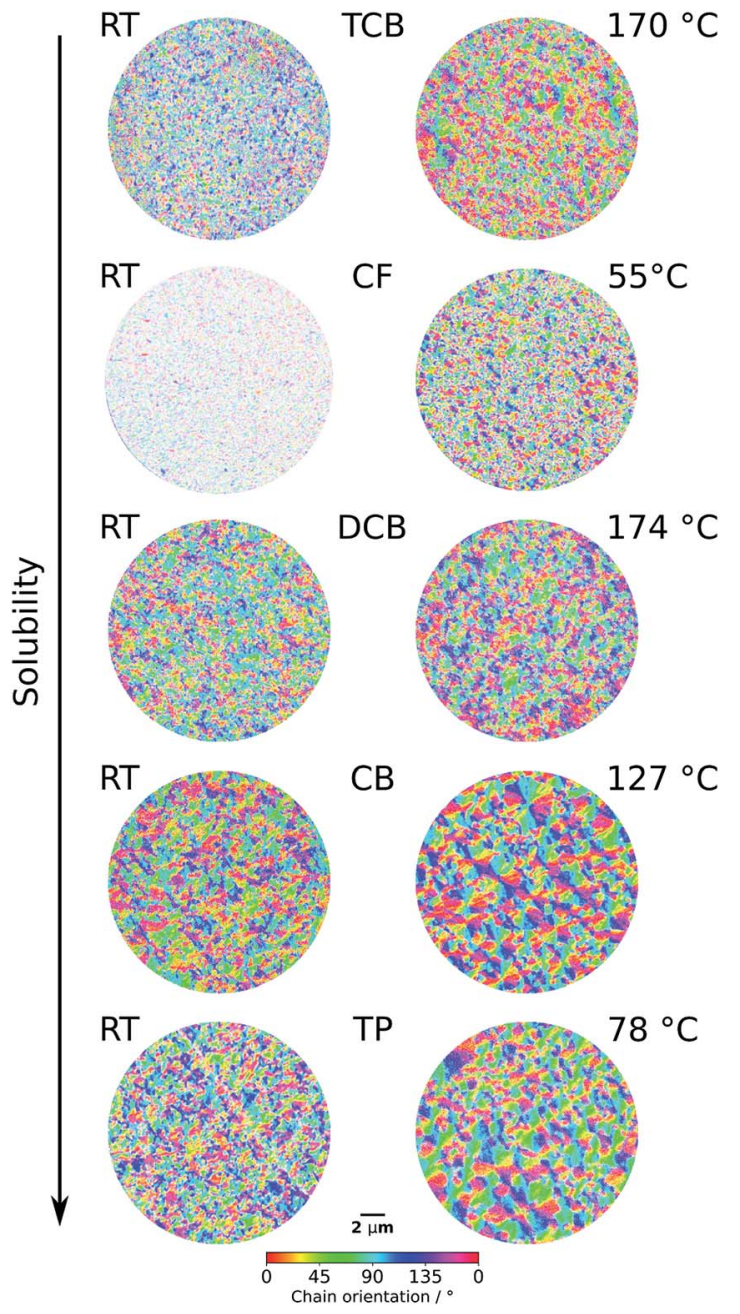

Fig. 1 Evaluated PEEM image series showing the combination of chain orientation and local degree of order of P3HT films spun from different solvents: CB, DCB, TCB, TP, CF. The left side shows the results for the films spun at room temperature, while the right hand side shows the results for the films spun from elevated temperatures (see Experimental section in the ESI for details $\uparrow$ ).

the OPL is an indirect measure for the domain size present in the organic film - the smaller the domains, the smaller the OPL.

Comparing the OPLs for each solvent (see Fig. S3†), we observe that the films spun from heated solutions exhibit larger domain sizes and higher degrees of order across all investigated solvents, except DCB. In the case of DCB the change in OPL were marginal.

The overall trend was expected as Machui et al. point out that the P3HT solubility is strongly temperature dependent. ${ }^{\mathbf{1 6}}$ The increased solubility at elevated temperature leads to a reduced amount of aggregated P3HT in solution. These P3HT aggregates are likely acting as seeds for the polymer crystallisation, therefore, the more aggregates are left in the solution the higher the number of individual domains is expected to be. Consequently, the average size of these domains is expected to be smaller compared to those domains originating from a film with less aggregates. 
Spun at RT

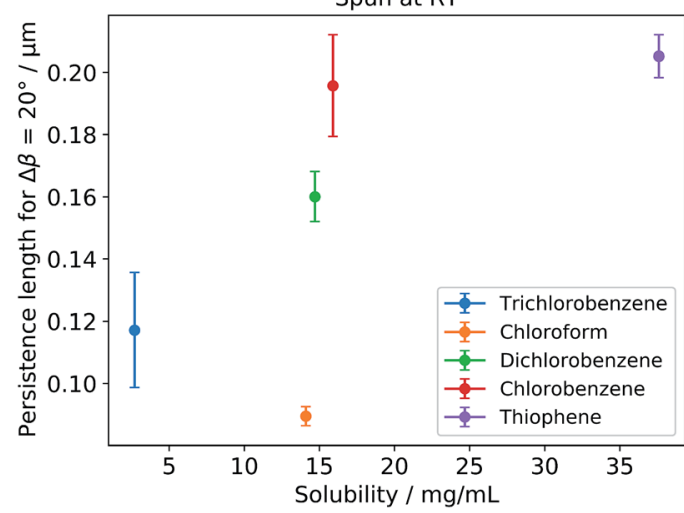

Fig. 2 Dependence of the OPL for $\Delta \beta=20^{\circ}$ plotted against the solubility of P3HT in different solvents. The values represent the mean value gathered from multiple spots on the same sample. The errorbars express the standard deviation (see ESI $\uparrow$ for more sample spots). We note that the value of $\Delta \beta=20^{\circ}$ has been chosen arbitrarily as a representation, however, comparing different values for $\Delta \beta$ (not shown) the chosen value is a valid representation.

Nevertheless, the results for DCB are unexpected. It can be deducted, that the increased solubility does not compensate for other effects that influence the domain size. These findings are supported by recent results form Roesing et al. ${ }^{18}$ They report that the self-assembly mechanisms and the solubility behavior are not equally determined by the same intermolecular interactions. However, as they do not investigate DCB in their report directly, caution should be exercised before reaching a final conclusion. Furthermore, we are confident that the experimental findings presented here stimulate further theoretical insights into the factors that govern the solvent effects on the polymer film structure.

In addition, we note that the higher degree of order and the larger domain size does not necessarily lead to a better device performance. The reason are "bridging chains" that can connect from one domain to a neighbouring one, thus acting as pathways for charge carriers. See the ESI $\uparrow$ for the properties of the P3HT employed here. ${ }^{19}$

The same statement, of multiple effects having a decisive impact on the morphology, can also be made when we transition films spun cast from one solvent at two different temperatures to films spun from different solvents at room temperature.

As previously mentioned, multiple proposals have been made in the literature to correlate one or more solvent properties with the resulting morphology or device performance. As for the boiling point, in case of the heated and non-heated solvents, the solvent with the highest boiling point (TCB) does not yield the film with the largest domains nor the highest degree of order (lowest amount of transparency). Furthermore, TCB is a special case: the solubility at RT is actually below the concentration used in this study. The incomplete dissolution of the P3HT leads to an inhomogeneous film, with no defined thickness (see ESI $\dagger$ ). In addition, the partial dissolution leads to a high number of seeds that in turn lead to smaller domains in the film. Again, this is in line with the proposal made by Yang et al. that other solvent parameters such as the solubility parameters and the difference in heat capacity should be considered in addition to the boiling point. ${ }^{\mathbf{1 4}}$

We note that a strong variation in film thickness has been observed for the samples presented in this report (see $\operatorname{ESI} \dagger$ ). However, no correlation between the domain size and the film thickness has been observed here, therefore it can be excluded as a parameter of interest.

Here, we propose to use the solubility of P3HT in the respective solvent as the primary indicator for the domain size. In Fig. 2 the OPL for each respective solvent is plotted against the P3HT solubility in each solvent. We observe a correlation of the OPL including di-, tri- and chlorobenzene as well as thiophene.

However, chloroform has to be considered separately, as it breaks with the trend. We assume that the reason for the rather small OPL, therefore also the small domain size, is the high vapor pressure of chloroform. This leads to very short drying times of the films, leaving the polymer chains less time to aggregate properly. Again, this stresses the point that a single discriminator seems to be insufficient to predict the domain size, or even the morphology of P3HT films.

In conclusion, we have obtained orientation maps of five different solvent/P3HT combinations at two different temperatures each. By comparing the images visually and with the help of the OPL method we were able to identify the solubility as the principal parameter determining the P3HT domain size. The boiling point that has been extensively discussed in literature, seems to play a secondary role only. However, we note that the PEEM technique employed in this report is highly surface sensitive, whereas many of the previously available methods yield information concerning the bulk structure. ${ }^{11}$ This fact is a possible explanation for the differences between the results presented here and the literature. In addition, we point out again that other groups have stressed the importance of the solubility as well. ${ }^{12,13}$

Taking all observations into account we again stress the point that the ability to investigate the surface morphology of different P3HT samples, especially with respect to the domain sizes and the grain boundaries, is a valuable tool to tackle challenges that relate to the surface morphology and function of semiconducting polymer films.

\section{Conflicts of interest}

There are no conflicts to declare.

\section{Acknowledgements}

The work was funded in part by the German Science Foundation (DFG) through SFB-TR102. F. N. gratefully acknowledges funding from the DBU. F. N., A. K. and B. A. thank APE Angewandte Physik und Elektronik GmbH for support with the Harmonixx. All authors are deeply indebted to Dr Katrin Siefermann and Dr Andreas Neff for their contributions in the early stages of this project. The authors thank Dr Mike Hambsch for insightful 
discussions concerning the film preparation. The authors acknowledge support by the European Regional Development Fund (ERDF).

\section{Notes and references}

1 J. Rockström, W. Steffen, K. Noone, S. Persson, F. S. Chapin, E. F. Lambin, T. M. Lenton, M. Scheffer, C. Folke, H. J. Schellnhuber, et al., Nature, 2009, 461, 472-475.

2 J. Rockström, H. J. Schellnhuber, B. Hoskins, V. Ramanathan, P. Schlosser, G. P. Brasseur, O. Gaffney, C. Nobre, M. Meinshausen, J. Rogelj and W. Lucht, Earth's Future, 2016, 4, 465-470.

3 J. Rockström, O. Gaffney, J. Rogelj, M. Meinshausen, N. Nakicenovic and H. J. Schellnhuber, Science, 2017, 355, 1269-1271.

4 W. Steffen, J. Rockström, K. Richardson, T. M. Lenton, C. Folke, D. Liverman, C. P. Summerhayes, A. D. Barnosky, S. E. Cornell, M. Crucifix, J. F. Donges, I. Fetzer, S. J. Lade, M. Scheffer, R. Winkelmann and H. J. Schellnhuber, Proc. Natl. Acad. Sci. U. S. A., 2018, 115, 8252-8259.

5 H. Zhou, L. Yang and W. You, Macromolecules, 2012, 45, 607632.

6 M. T. Dang, L. Hirsch and G. Wantz, Adv. Mater., 2011, 23, 3597-3602.

7 C. R. McNeill, J. Polym. Sci., Part B: Polym. Phys., 2011, 49, 909-919.

8 B. A. Collins, J. E. Cochran, H. Yan, E. Gann, C. Hub, R. Fink, C. Wang, T. Schuettfort, C. R. McNeill, M. L. Chabinyc and H. Ade, Nat. Mater., 2012, 11, 536-543.
9 S. Kuehn, P. Pingel, M. Breusing, T. Fischer, J. Stumpe, D. Neher and T. Elsaesser, Adv. Funct. Mater., 2011, 21, 860-868.

10 E. Klimov, W. Li, X. Yang, G. G. Hoffmann and J. Loos, Macromolecules, 2006, 39, 4493-4496.

11 A. Neff, F. Niefind, B. Abel, S. C. B. Mannsfeld and K. R. Siefermann, Adv. Mater., 2017, 1701012.

12 Z. Bao, A. Dodabalapur and A. J. Lovinger, Appl. Phys. Lett., 1996, 69, 4108-4110.

13 J.-F. Chang, B. Sun, D. W. Breiby, M. M. Nielsen, T. I. Sölling, M. Giles, I. McCulloch and H. Sirringhaus, Chem. Mater., 2004, 16, 4772-4776.

14 H. Yang, T. J. Shin, L. Yang, K. Cho, C. Y. Ryu and Z. Bao, Adv. Funct. Mater., 2005, 15, 671-676.

15 M. T. Dang, G. Wantz, H. Bejbouji, M. Urien, O. J. Dautel, L. Vignau and L. Hirsch, Sol. Energy Mater. Sol. Cells, 2011, 95, 3408-3418.

16 F. Machui, S. Langner, X. Zhu, S. Abbott and C. J. Brabec, Sol. Energy Mater. Sol. Cells, 2012, 100, 138-146.

17 L. Chang, H. W. A. Lademann, J.-B. Bonekamp, K. Meerholz and A. J. Moulé, Adv. Funct. Mater., 2011, 21, 1779-1787.

18 M. Roesing, J. Howell and D. Boucher, J. Polym. Sci., Part B: Polym. Phys., 2017, 55, 1075-1087.

19 R. J. Kline, M. D. McGehee, E. N. Kadnikova, J. Liu, J. M. J. Fréchet and M. F. Toney, Macromolecules, 2005, 38, 3312-3319. 